Research Paper

\title{
The Effectiveness of Ropivacaine and Mepivacaine in the Postoperative Pain after Third Lower Molar Surgery
}

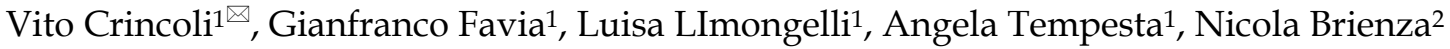 \\ 1. Interdisciplinary Department of Medicine, University of Bari, Italy. \\ 2. Anesthesia and Intensive Care Unit, Department of Emergency and Organ Transplantation, University of Bari, Italy. \\ $\triangle$ Corresponding author: Prof. Vito Crincoli, Interdisciplinary Department of Medicine, University of Bari, Italy; Piazza Giulio Cesare 11, 70124 Bari, Italy. E \\ mail: vito.crincoli@uniba.it; Phone: 00390805478051 - Fax 00390805478743 \\ (C) 2015 Ivyspring International Publisher. Reproduction is permitted for personal, noncommercial use, provided that the article is in whole, unmodified, and properly cited. \\ See http://ivyspring.com/terms for terms and conditions.
}

Received: 2015.06.28; Accepted: 2015.09.20; Published: 2015.10.16

\begin{abstract}
Aim: To compare the efficacy of $0.75 \%$ ropivacaine with $3 \%$ mepivacaine for pain control in the first 24 hours after surgical removal of lower third molars, using a quantitative measurement such as VAS. The secondary objective involved rescue analgesia.

Methods: Forty-five patients, 21 females and 24 males, mean age 23,2 \pm 3 years, underwent surgical removal of third molars in two separate sessions. A split-mouth design was chosen, so each patient underwent both the first and second surgeries, having for each extraction a different anesthetic. The second extraction was carried out 1 month later. Parameters evaluated were: onset of anesthesia, duration of surgery, lip numbness, timing of pain appearance and first analgesic intake.

Results: No significant differences about onset of anesthesia, duration of surgical procedures, and timing of first analgesic intake were found. Lower lip numbness, on the other hand, was more prolonged after using ropivacaine $(p<0.0001)$ and the onset of postoperative pain was more delayed after anesthesia with ropivacaine $(p=0.0048)$. Pain scores at 1 and 2 hours after surgery were $3.5 \pm 2.0$ and $4.1 \pm 1.3$ after injection of mepivacaine, and $2.7 \pm 2.2$ and $2.9 \pm 2.4$ after ropivacaine ( $p$ value $=0.006$ for both time points). No significant differences in pain score were recorded between the two anesthetics at 12 and 24 hours post surgery.

Conclusions: With the use of ropivacaine, the discomfort caused by prolonged lip numbness is counterbalanced by less postoperative discomfort after surgery. In addition, when compared with other long-acting anesthetics, ropivacaine ensures a safer anesthetic profile for medically complex patients.
\end{abstract}

Key words: local anesthetics, third molar surgery, postoperative pain.

\section{Introduction}

Local anesthesia for dental surgery is usually done with rather short-lasting and safe drugs, such as mepivacaine or articaine. The idea that using long-lasting local anesthetics, such as bupivacaine or its safer derivatives ropivacaine and levobupivacaine, would improve the quality of care after removal of mandibular third molars is based on the fact that a part of postoperative time would be covered by residual effects of anesthesia, thus reducing pain or analgesics consumption. However, such advantages would be counterbalanced by a greater risk of toxicity
[1]. Ropivacaine is a new long-acting enantiomerically pure (S-enantiomer) amide local anesthetic, structurally related to bupivacaine, but with less cardiac toxicity and neurotoxicity [2]. Available data show ropivacaine to be very suitable for regional anesthesia. It has been tested in dentistry with encouraging results about its duration of action [3]. In a recent randomized clinical trial, ropivacaine was compared with lignocaine hydrochloride for lower third molar extractions [4]. The primary objective of the present study was to compare the efficacy of $0.75 \%$ ropiva- 
caine with 3\% mepivacaine, one of the most common anesthetics used in dentistry, for pain control in the first 24 hours after surgery, using a quantitative measurement such as VAS; the secondary objective involved rescue analgesia.

\section{Materials and Methods}

This prospective, randomized, controlled study was performed from January 2014 to November 2014 at the School of Dentistry, University of Bari, Italy, in accordance with the provisions of the Declaration of Helsinki. Institutional human experimentation panel approval and informed consent from each patient were obtained. Patients requiring bilateral surgical removal of impacted third lower molars were recruited. Age $>18$ years or $<40$ years, and good health, as assessed by patients' history, clinical examination, blood pressure and pulse rate, were inclusion criteria. Moreover, depth of impaction, angulation and relationship of lower third molars with the mandibular branch had to be overlapping on both sides [5]. Exclusion criteria were: age $<18$ years or $>40$ years, lower third molars with different characteristics and indications for extraction, systemic diseases such as immunosuppression, diabetes mellitus, cardiovascular or liver diseases, smoking, pregnancy, allergy to beta-lactam antibiotics and local anesthetics, consumption of antibiotics or anti-inflammatory drugs that would have altered pain perception in the 2 weeks prior to the surgical procedure, clinical signs of infection and inflammation around lower third molars at the time of surgery, presence of anxiety requiring the use of sedative or anxiolytic drugs. All extractions were carried out without premedication.

Forty-five patients, 21 females and 24 males (mean age 23,2 \pm 3 years) were successfully enrolled for the study. They underwent two surgical procedures, for a total of 90 partially impacted lower third molars extracted. Random sampling by means of sealed envelopes was used to determine which of the two molars (right or left) should have been extracted first and which anesthetic (mepivacaine or ropivacaine) should have been used for the first extraction. The second extraction was carried out a month later using the other anesthetic. This "wash out" period spaced out the two surgeries in order to eliminate the effects of the first drug, thus bringing the patient in the initial conditions [5]. Each patient was blind to the type of anesthetic used. All the extractions were performed by a single experienced practitioner with a standard technique: disinfection of the surgical site with iodine, alveolar nerve block, infiltration of buccal soft tissues, incision of triangular mucoperiosteal flap, corticotomy by water-cooled burr in a surgical drill, odontotomy by water-cooled burr, tooth extraction by straight or Barry lever, surgical curettage by Volkmann spoon, irrigation of post-extraction sites with saline solution and 3/0 polyglactin (Vicryl) interrupted sutures. For the molar extraction with ropivacaine, the alveolar nerve block was performed by means of $2 \mathrm{~mL}$ solution $(7.5 \mathrm{mg} / \mathrm{ml}$ Naropin $\mathrm{TM}^{\circledR}$ AstraZeneca, Sweden), and buccal soft tissues were infiltrated with $1.0 \mathrm{~mL}$ of the same solution, taken by a $10 \mathrm{ml}$ vial of ropivacaine. In the mepivacaine (Carbosen) group, the alveolar nerve block was obtained by means of $1.8 \mathrm{ml}$ solution $(30 \mathrm{mg} / \mathrm{ml})$ without epinephrine. The buccal soft tissues were anesthetized with $1.8 \mathrm{ml}$ solution $(20 \mathrm{mg} / \mathrm{ml})$ of the same local anesthetic with 1:80.000 epinephrine [6].

The different concentrations employed of ropivacaine and mepivacaine were calculated in order to obtain an equivalence of power. Besides, epinephrine was added in the mepivacaine group for soft tissue anesthesia for ethical reason, considering that ropivacaine has intrinsic vasocontrictive properties.

Each patient was prescribed oral post-surgical antibiotic therapy (Amoxicillin + clavulanic acid $1 \mathrm{~g}$ tablets twice a day for 6 days). In case of pain, ibuprofen $600 \mathrm{mg}$ was suggested.

In the intraoperative period, the following parameters were evaluated: 1 ) onset of anesthesia (the period between the end of the local anesthetic administration and the onset of lower lip anesthesia); 2) duration of surgery (from incision to wound closure).

Once discharged, patients were asked by a single person blind to the anesthetic used to complete a form reporting: 1) postoperative pain intensity on a visual anagogic scale (VAS) [7] at 1, 2, 12 and 24 hours after surgery, 2) duration of lower lip numbness by lightly tapping the lower lip with index or middle finger, 3 ) time lapse to postoperative pain, 4) time lapse to first analgesic intake, 5) any adverse effect (Figure 1).

To compare the effectiveness of the two different anesthetics in pain control, a "split-mouth" design was chosen [8,9], where each of two treatments is randomly assigned to either the right or left halves of the same patient's dentition. By making within-patient comparisons, rather than between-patient comparison, the error variance (noise) of the experiment can be reduced, thereby obtaining a more powerful statistical test. The advantage of the design is that it removes a lot of inter-individual variability from the estimates of the treatment effect.

\section{Statistical analysis}

A pilot study with mepivacaine indicated that 45 patients were necessary in order for the trial to have an $80 \%$ power of detecting a 1-point decrease in VAS with ropivacaine, at $\mathrm{a}=0.05$. 
Questionnaire reserved to the patient

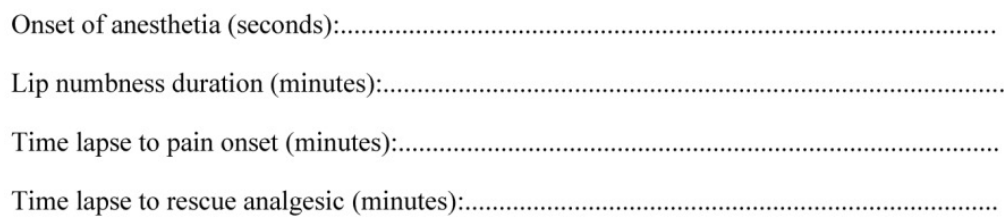

Pain evaluation on a VAS scale at 1, 2, 12, 24 hours post-surgery:

\section{Visual Analogue Scale (VAS)}

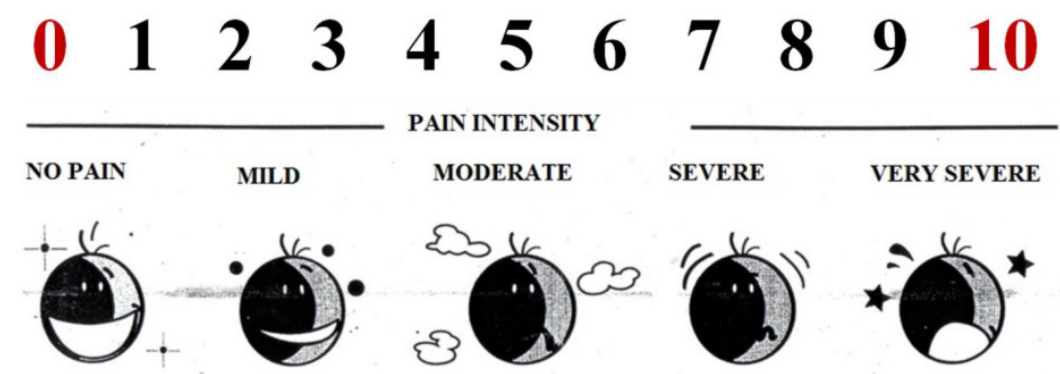

Figure 1. Postoperative patients form.

Data regarding lip numbness, timing of pain appearance and analgesic consumption were converted into minutes; onset time was expressed in seconds.

Continuous normally distributed data were expressed as mean \pm standard deviation (SD) and compared using paired Student $t$ test. Non-normally distributed data were expressed as median and interquartile ranges and compared using the Wilcoxon test for paired samples. Categorical data were expressed as number and percentage and compared using chi-squared $\left(X^{2}\right)$ or the Fisher's exact tests. The effects over time of the two anesthetics on pain intensity (i.e., VAS) were evaluated by Analysis of variance (ANOVA). A Newman-Keuls post-hoc test was performed to detect any significant difference between groups. In all comparisons, a $p$ value $\leq 0.05$ was considered statistically significant.

\section{Results}

Table 1 shows data about onset of anesthesia, duration of surgery, lower lip numbness duration, postoperative pain onset and time of first requirement of analgesics.

Data about onset gave no statistically significant differences. Lower lip numbness, on the other hand, was more prolonged after using ropivacaine ( $p$ value $<0.0001)$. In three cases, onset of postoperative pain was not recorded. Therefore analysis was performed only in 42 patients. Onset of postoperative pain was more delayed after anesthesia with ropivacaine $(p$ value $=0.0048$ ). There was no significant difference in timing of first analgesic intake between the two surgical sessions. Pain scores at 1 and 2 hours after surgery were $3.5 \pm 2.0$ and $4.1 \pm 1.3$ after injection of mepivacaine, and $2.7 \pm 2.2$ and $2.9 \pm 2.4$ after ropivacaine ( $p$ value $=0.006$ for both time points). No significant differences in pain score were recorded between the two anesthetics at 12 and 24 hours post surgery (Figure 2). No side effect was reported with either mepivacaine or ropivacaine.

Table 1. Onset of Anesthesia, Duration of Surgery, Duration of Lip Numbness, Delay to Pain Onset, Delay to Rescue Analgesic Intake.

\begin{tabular}{|c|c|c|c|}
\hline & $\begin{array}{l}\text { Mepivacaine } \\
\text { anaesthesia } \\
(\mathrm{N}=45)\end{array}$ & $\begin{array}{l}\text { Ropivacaine } \\
\text { anaesthesia } \\
(\mathrm{N}=45)\end{array}$ & $p$ value \\
\hline $\begin{array}{l}\text { Onset of anaesthesia (sec) } \\
\text { Median (Quartile) }\end{array}$ & $120(62-173)$ & $140(60-152)$ & ns \\
\hline $\begin{array}{l}\text { Surgery duration (min) } \\
\text { Mean } \pm \text { SD }\end{array}$ & $19.8 \pm 7$ & $21.9 \pm 8.4$ & ns \\
\hline $\begin{array}{l}\text { Lip numbness duration (min) } \\
\text { Median (Quartile) }\end{array}$ & $169(157-182)$ & $409(266-496)$ & $<0.0001$ \\
\hline $\begin{array}{l}\text { Time lapse to pain onset (min)* } \\
\text { Median (Quartile) }\end{array}$ & $154(75-194)$ & $173(123-330)$ & 0.0048 \\
\hline $\begin{array}{l}\text { Time lapse to rescue analgesic } \\
\text { (min) } \\
\text { Median (Quartile) }\end{array}$ & $333(110-569)$ & $357(127-498)$ & ns \\
\hline
\end{tabular}




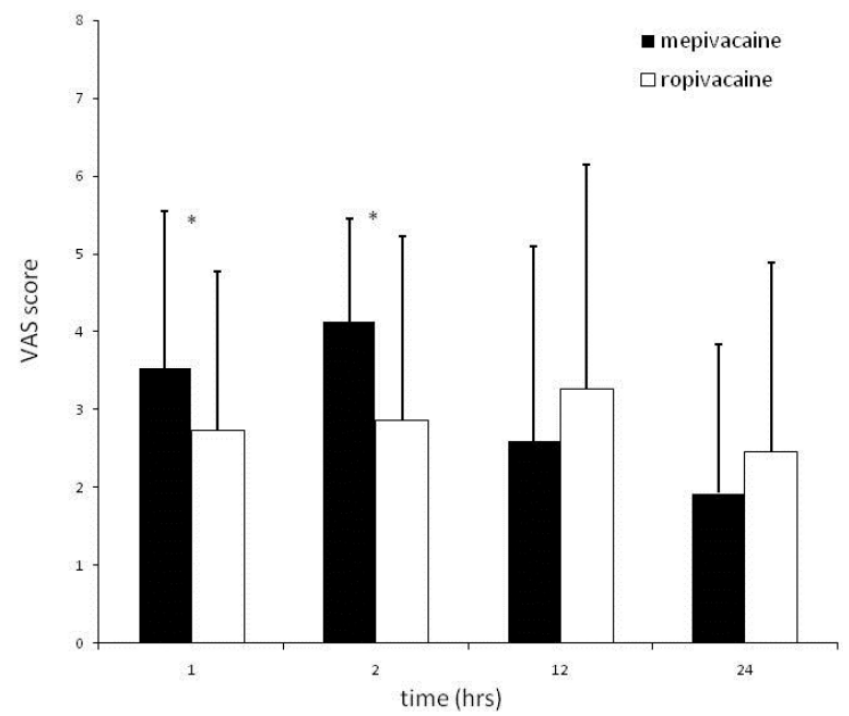

Figure 2. Mean ( \pm SD) VAS scores at 1, 2, 12 and 24 hours after surgery in mepivacaine (full bars) and ropivacaine (empty bars) groups. $*=p<0.05$ between groups.

\section{Discussion}

Long-acting local anesthetic agents are suggested in lengthy dental treatments for control of post-surgical pain. Although the available anesthetics for dentistry have minimal side effects in the doses usually employed, they might cause potential problems. Bupivacaine, for example, shows toxic effects on the central nervous and cardio-vascular systems, while etidocaine may affect intraoperative bleeding. Ropivacaine appears to offer advantages over either of the currently used long-acting agents [10].

In the present trial, the comparison between ropivacaine and mepivacaine regarding the onset time showed no significant differences. El-Sharrawy et al. [11] stated that an increased concentration of ropivacaine reduces the onset time of peripheral nerve block probably due to the higher amount of molecules able to penetrate the nerve per unit time. On the contrary, the ropivacaine solution did provide a long-acting anesthetic effect as demonstrated by the longer duration of lower lip numbness when compared to mepivacaine. This observation is in line with a previous study [12] reporting more than 4 hours of lip numbness and a complete numbness and tingling recovery after 6 hours with ropivacaine in dental surgery. This result may be related to ropivacaine's intrinsic characteristics. Its low liposolubility blocks nerve fibers involved in pain transmission (thin $\mathrm{A} \delta$ and $C$ fibers) to a greater degree than those controlling motor function (large $A \beta$ fibers). Unlike most local anesthetics, which are vasodilatators, ropivacaine produces vasoconstriction in vitro and in vivo in animal models $[13,14]$.The vasoconstrictive properties and strong bond to plasma proteins prolong anesthesia duration. Specifically, the prolonged duration of lip numbness was the most significant differentiator of ropivacaine. However, this effect can cause difficulty in eating, drinking and speaking and inadvertent biting of the lips. Kennedy and coworkers [12] observed that the duration of pulpal anesthesia was shorter for ropivacaine without epinephrine, suggesting the use of adrenaline to prolong the duration of soft tissue anesthesia induced by ropivacaine. However, in the present study, ropivacaine, even without epinephrine, when compared with mepivacaine with epinephrine, achieved successful postoperative pain control in the first two hours after dental extractions. The absence of significant differences in pain intensity in the following VAS evaluations (12 and 24 hours) may be related to the use of systemic analgesics. As a matter of fact, the time lapse to rescue analgesic was similar between mepivacaine and ropivacaine. This result differs from that of a previous study [6], in which the use of levobupivacaine was associated to both significantly longer analgesia duration and time lapse to rescue analgesic when compared to mepivacaine. Enberg and coworkers [15] enrolled 30 healthy individuals who received a ropivacaine injection in three randomized concentrations $(2.0,5.0$ or $7.5 \mathrm{mg} / \mathrm{ml})$ for infiltration anesthesia and mandibular nerve block. They showed that only ropivacaine at $7.5 \mathrm{mg} / \mathrm{ml}$ produced a long duration of both pulpal and soft tissue anesthesia, which may be helpful in control of post-operative pain. El-Sharrawy et al. [11] tested the anesthetic efficacy of different ropivacaine concentrations $(0.75 \%, 0.5 \%, 0.375 \%$ or $0.25 \%$ ) for inferior alveolar nerve block. El-Sharrawy's data underlined that $0.5 \%$ and $0.75 \%$ ropivacaine concentrations were effective for alveolar nerve block and produced a rapid onset and a prolonged pain control. Buric [16] used $0.75 \%$ of ropivacaine for infiltrative anesthesia in eight patients undergoing upper and lower third molar extractions, cystectomy, apicoectomy and other tooth extractions and concluded that this concentration enabled successful and long-acting local anesthesia. Brkovic et al [17] assessed the clinical efficacy and hemodynamic effects of ropivacaine. The postoperative need for analgesics was observed in $67-100 \%$ of patients. Meechan [18] compared the efficacy of 0.75 and $1 \%$ ropivacaine with $2 \%$ lidocaine with 1:80,000 epinephrine for intraligamentary anesthesia of upper lateral incisors. The author concluded that lidocaine with epinephrine was more successful than ropivacaine in the tested concentrations in obtaining pulpal anesthesia. Considering the previously cited studies, we also have used ropivacaine in the $0.75 \%$ concentration, which 
seems to be the most effective. A split-mouth study design $[8,9]$ has been chosen to reset all bias. Sample size is the main problem with this protocol, due to the difficulty in finding similar characteristics in collaborating patients. Nevertheless, all the misleading factors linked to the characteristics of the subjects are eliminated, ensuring that the test is accurate and that the results are viable and conclusive.

The results of this trial show that while ropivacaine has residual effects in the early postoperative time, it has a weak effect on the overall quality of analgesia, as no significant differences in pain score were recorded between the two groups at 12 and 24 hours post-surgery.

\section{Conclusions}

With the use of ropivacaine, the discomfort caused by prolonged lip numbness is counterbalanced by less postoperative discomfort after extraction of third lower molars. In addition, when compared with other long-acting anesthetics, ropivacaine ensures a safer anesthetic profile for medically complex patients.

Special thanks to Dr. Maria Massari for her assistance.

\section{Competing Interests}

The authors have declared that no competing interest exists.

\section{References}

1. Sisk AL. Long-acting local anesthetics in dentistry. Anesth Prog. 1992;39:53-60.

2. Hansen TG. Ropivacaine: a pharmacological review. Expert Review of Neurother. 2004;4:781-91.

3. Davis MJ, Vogel LD. Local anesthetic safety in pediatric patients. N Y State Dent J. 1996;62:2-35.

4. Budharapu A, Sinha R, Uppada UK, Subramanya Kumar AV. Ropivacaine: a new local anaesthetic agent in maxillofacial surgery. Br J Oral Maxillofac Surg. 2015 May;53:451-4.

5. Crincoli V, Di Comite M, Di Bisceglie MB, Petruzzi M, Fatone L, De Biase C, Tecco S, Festa F. Which route of antibiotic administration should be used for third molar surgery? A split-mouth study to compare intramuscular and oral intake. Clin Ter. 2014;165:e12-6.

6. Crincoli V, Di Bisceglie MB, Massaro M, Giuliani R, Favia G, Brienza N. Postoperative pain relief after surgical removal of impacted third molars: a single-blind,randomized, controlled study to compare levobupivacaine and mepivacaine. J Orofac Pain. 2009;23:325-9.

7. Tuffin JR, Cunliffe DR, Shaw SR. Do local analgesics injected at the time of third removal under general anaesthesia reduce significantly post operative analgesics requirements? A double-blind controlled trial. Br J Oral Maxillofac Surg. 1989;27:27-32.

8. Antczak-Bouckoms AA, Tulloch JF, Berkey CS. Split-mouth and cross-over designs in dental research. J Clin Periodontol 1990; 17:446-53.

9. Hujoel PP, DeRouen TA. Validity issues in split-mouth trials. J Clin Periodontol 1992;19:625-7.

10. Akerman B, Hellberg IB, Trossvik C. Primary evaluation of the local anaesthetic properties of the amino amide agent ropivacaine (LEA 103). Acta Anaesthesiol Scand. 1988;32:571-8.

11. El-Sharrawy E, Yagiela JA. Anesthetic efficacy of different ropivacaine concentrations for inferior alveolar nerve block. Anesth Prog. 2006;53:3-7.

12. Kennedy M, Reader A, Beck M, Weaver J. Anesthetic efficacy of ropivacaine in maxillary anterior infiltration. Oral Surg Oral Med Oral Pathol Oral Radiol Endod. 2001;91:406-12.

13. Iida $\mathrm{H}$, Ohata $\mathrm{H}$, Iida $\mathrm{M}$, et al. The differential effects of stereoisomers of ropivacaine and bupivacaine on cerebral pial arterioles in dogs. Anesth Analg. 2001;93:1552-6.
14. Timponi CF, Oliveira NE, Arruda RM, Meyrelles SS, Vasquez EC. Effects of the local anaesthetic ropivacaine on vascular reactivity in the mouse perfused mesenteric arteries. Basic Clin Pharmacol Toxicol. 2006;98:518-20.

15. Enberg M, Kopp S. Ropivacaine for dental anesthesia: a dose-finding study. J Oral Maxillofac Surg. 2002;60:1004-10.

16. Buric N. The assessment of anesthetic efficacy of ropivacaine in oral surgery. N Y State Dent J. 2006; 72:36-9.

17. Brkovic BM, Zlatkovic M, Jovanovic D, Stojic D. Maxillary infiltration anaesthesia by ropivacaine for upper third molar surgery. Int J Oral Maxillofac Surg. 2010;39:36-41.

18. Meechan JG. A comparison of ropivacaine and lidocaine with epinephrine for intraligamentary anesthesia. Oral Surg Oral Med Oral Pathol Oral Radiol Endod. 2002;93:469-73. 